Meta

Journal des traducteurs

Translators' Journal

\title{
Index du volume 30
}

Volume 30, numéro 4, décembre 1985

URI : https://id.erudit.org/iderudit/003179ar

DOI : https://doi.org/10.7202/003179ar

Aller au sommaire du numéro

Éditeur(s)

Les Presses de l'Université de Montréal

ISSN

0026-0452 (imprimé)

1492-1421 (numérique)

Découvrir la revue

Citer ce document

(1985). Index du volume 30. Meta, 30(4), 408-423.

https://doi.org/10.7202/003179ar

Ce document est protégé par la loi sur le droit d'auteur. L'utilisation des services d'Érudit (y compris la reproduction) est assujettie à sa politique d'utilisation que vous pouvez consulter en ligne.

https://apropos.erudit.org/fr/usagers/politique-dutilisation/ 


\section{Index \\ du \\ volume 30}

\section{A. ARTICLES}

Apter, R. : A Peculiar Burden : Some Technical Problems of Translating Opera for Performance into English, no 4, p. 309.

Bathgate, R. H. : Studies of Translation Models 3, $n^{\circ} 3$, p. 129.

Berman, A. : La traduction et la langue française, $n^{\circ} 4$, p. 341 .

Bowen, D. \& M. : The Nuremberg Trials, $n^{\circ} 3$, p. 74.

Bühler, H. : Conference Interpreting, $n^{\circ} 1$, p. 49.

Daly, A. : Interpreting for International Satellite Television, $\mathrm{n}^{0} 1$, p. 91.

Déjean le Féal, K. : Le registre littéraire en interprétation simultanée, $\mathrm{n}^{\circ} 1$, p. 55.

Garcia-Landa, M. : L'oralité de la traduction orale, $\mathrm{n}^{\circ} 1$, p. 30.

Gémar, J.-C. : Réflexions sur la manière de traduire, $n^{0} 3$, p. 236.

Gile, D. : Le modèle d'efforts et l'équilibre d'interprétation en interprétation simultanée, $n^{\circ} 1$, p. 44.

Gile, D. : De l'idée à l'énoncé, no 2 , p. 139.

Gile, D. : Les termes techniques en interprétation simultanée, no 3, p. 199.

Gile, D. : L'interprétation de conférence et la connaissance des langues, no 4 , p. 320.

Gouadec, B. : Microdictionnaire avec micro-encyclopédie et illustration par diapositives pilotées, $\mathrm{n}^{\circ} 4$, p. 332.

Ilg, G. : Expressions, no 1, p. 65 .

Jensen, P. A. : SI : A Note on Error Typologies and the Possibility of Gaining Insight in Mental Processes, $\mathrm{n}^{\circ} 1, \mathrm{p} .106$.

Jumpelt, R. W. : The Conference Interpreter's Working Environment under the New ISO and IEC Standards, $n^{\circ} 1$, p. 82.

Kelkar, A. R. : To Translate or not to Translate ?, no 3, p. 211.

Kocourek, R. : Terminologie et efficacité de la communication, $n^{\circ} 2$, p. 119.

Lambert, S.:M. \& W. E. : Physiology : a Questionnaire, $\mathrm{n}^{\circ}$ 1, p. 68.

Lederer, M. : L'interprétation, manifestation élémentaire de la traduction, $\mathrm{n}^{\circ}$ 1, p. 25.

Le Meur, A. : Microdictionnaire avec micro-encyclopédie et illustration par diapositives pilotées, $n^{\circ} 4$, p. 332.

Mackintosh, J. : The Kintsch and Van Dijk Model of Discourse, $n^{\circ} 1$, p. 37.

Marian Marica, I. : Light Relief A Palindromic Interpreter's Life, $\mathfrak{n}^{\circ} 1$, p. 73.

Moser, W. : Les pulsations de la traduction, $n^{\circ} 1$, p. 7.

Moser-Mercer, B. : Screening Potential Interpreters, $n^{\circ} 1$, p. 97.

Orléans-Gerstein, É. : Un mot...vaut-il mille images ?, $n^{\circ} 1$, p. 5.

Romer, Th. : Le métier d'interprète - d'hier à demain, $\mathrm{n}^{\circ}$ 1, p. 101.

Seleskovitch, D. : Interprétation ou interprétariat ?, no 1 , p. 19.

Schweda-Nicholson, N. : Consecutive Interpretation Training, $\mathrm{n}^{\circ} 2$, p. 148.

Thiéry, Ch. : La responsabilité de l'interprète de conférence professionnel, $\mathrm{n}^{\circ} 1$, p. 78.

Wilss, W. : Rhetorical and Stylistic Issues in Translation Pedagogy, no 3, p. 236. 


\section{B. ÉTUDES TERMINOLOGIQUES ET LINGUISTIQUES}

Bagge, Ch. : Doyen ou doyenne, $n^{\circ} 3$, p. 255.

Cormier, M. C. : Glossaire de la théorie interprétative de la traduction et de l'interprétation, $n^{\circ} 4$, p. 353.

Dupont, Ch. : Commuter : un mot pour tout dire, $n^{\circ} 4$, p. 379.

Duquet Picard, D. : Normalisation et néonymie en français moderne, $n^{\circ} 3$, p. 257.

Laurian, A.-M. : Informatique, traduction et enseignement des langues, $n^{\circ} 3$, p. 274.

Lavoie, A.-M. : Les bovins de boucherie, $n^{\circ} 4$, p. 359.

Lépinette Lepers, B. : Séries lexicalisées et dictionnaires bilingues, $n^{\circ} 3$, p. 242.

Lethuillier, J. : Jusqu'au diesel, dans l'arbre des moteurs thermiques, $\mathrm{n}^{\circ}$ 3, p. 261.

Paul, S. Terminologie de la télédétection aérospatiale, $n^{\circ} 4$, p. 364 .

Quillard, G. : Je ne file pas très bien aujourd'hui ou Comment ne pas traduire le verbe to feel, $\mathrm{n}^{\circ} 2, \mathrm{p} .268$.

Roberts, R. : The Terminology of Translation, $n^{\circ} 4$, p. 344.

Sieny, M. E. : Scientific Terminology in the Arab World, $\mathrm{n}^{\circ} 2$, p. 155.

Sopena Balordi, A. : Séries lexicalisées et dictionnaires bilingues, $\mathrm{n}^{\circ} 3$, p. 242.

Zafio, M.N. : L'arbre de domaine en terminologie, $\mathrm{n}^{\circ} 2$, p. 161.

\section{DOCUMENTATION}

Serré, R. : Ouvrages récents qui englobent un lexique ou un glossaire, $n^{\circ} 2$, p. $176 ; n^{\circ} 3$, p. $281 ; n^{\circ} 4$, p. 386. Spilka, I. V. : Bibliographie, $n^{\circ} 2$, p. $174 ; n^{\circ} 3$, p. $280 ; n^{\circ} 4$, p. 385.

\section{COMPTES RENDUS}

Bélanger, G. : Thésaurus multilingue - Économie de l'énergie, $\mathrm{n}^{\circ} 2, \mathrm{p} .181$. Brunet, J.-P. : Ca mange pas de pain !, $n^{\circ} 3$, p. 288.

Chezet, J. de : Dictionnaire des vrais amis, $n^{\circ} 2$, p. 184.

Clas, A. : Petit Larousse illustré 1985, $n^{\circ} 3$, p. 282.

Clas, A. : Elsevier's Dictionary of Jewellery and Watchmaking, $n^{\circ} 4$, p. 396.

Dandonneau, A. : Langage du droit et traduction, $n^{\circ} 2$, p. 182.

Faribault, M. : Introduction à la terminologie, $\mathbf{n}^{\circ} 2$, p. 179.

Faribault, M. : Rédaction technique, no 3, p. 288

Gianini, N. É. : Dictionnaire aérospatial, $n^{\circ} 4$, p. 398.

Gile, D. : Pathologie des fautes et maladresses dans la traduction, $n^{\circ} 2$, p. 177.

Guertin, F. : Dictionnaire aérospatial, no 4, p. 398.

Hosington, B. : Thomas Corneille's Ariadne Recreated in English, nº 3, p. 291.

Kully, R. : Österreichische Literatur in Übersetzungen, no 4, p. 397.

Martel, C. : Dictionnaire aérospatial, no 4, p. 398.

Nekrassoff, V. N. : Dictionary of Medicine, $n^{\circ}$ 3, p. 285.

Nekrassoff, V. N. : Data Systems Dictionary, $n^{\circ} 4$, p. 397.

Nespoulous, J.-L. : Glossogenetics : The Origin and Evolution of Language, $n^{\circ}$ 3, p. 284.

Nespoulous, J.-L. : Le discours physique du langage, $n^{\circ} 4$, p. 395.

Schwab, W. : Les trois états de la politique linguistique du Québec, no 3, p. 289.

Scutin, É. : Cuadernos de Traducción et Interpretación, no 4, p. 387.

Simon, Sh. : L'épreuve de l'étranger, no 4, p. 393.

Slote, D. : The Creative Writers Handbook, no 3, p. 287. 


\section{E. BLOC NOTES}

Cammaert, G. : Le traducteur et le droit d'auteur, $n^{\circ} 4$, p. 400.

Gentile, A. : Interpreting/Translating in Australia, $n^{\circ} 2$, p. 187.

Henry, R. : The Evolution of Laurentian University's Undergraduate Program for Translators and Interpreters, $\mathrm{n}^{\mathrm{o}} 3$, p. 300.

Juhel, D. : La place de la réflexion théorique dans l'apprentissage de la traduction, $n^{\circ}$ 3, p. 294.

Larose, R. : La théorie de la traduction : à quoi ça sert ?, nº 4, p. 405.

Lessard, D. : Humour - L'esprit des lois, no 4, p. 405.

Mareschal, G. : Colloque sur l'enseignement de la traduction juridique, $\mathrm{n}^{\circ} 4$, p. 407.

Roberts, R. : Evolution in Translation since 1966 as Reflected in the pages of META, no 2, p. 194.

Séguinot, C. : Translating Implication, $n^{\circ} 3$, p. 297.

Sudgen, D. : Machine Aids to Translation : Automated Language Processing System (ALPS), $n^{\circ} 4$, p. 403. 


\section{Index français des mots et des sujets traités}

Abattage, moment d', no 4, p. 362.

Abattage, rendement à l', $n^{\circ} 4$, p. 361

Acceptabilité, critère d', $\mathrm{n}^{\circ} 2$, p. 120.

Acception, no 4, p. 350.

Accoupler, n० 4, p. 360

Acte illocutoire, no 2, p. 142.

Activité traduisante, $n^{\circ} 3$, p. 239

Actualisation, $\mathrm{n}^{\circ} 1$, p. 22.

Actualisation sémique, $\mathrm{n}^{\circ} 1$, p. 22.

Adaptation, course à, no 3, p. 274.

Adiabatique, no 3, p. 262.

Affichage en couleur, no 4 , p. 365 .

Afficher, $n^{\circ} 4$, p. 365 .

Alimentation complémentaire, n० 4 , p. 360 .

Alimentation, frais d', $n^{\circ} 4$, p. 361 .

Aliments, no 4 , p. 361 .

Allumage commandé, moteur à, no 3, p. 272.

Allumage par compression, moteur à, no 3, p. 271

Allumage par étincelle, moteur à, no 3, p. 272.

ALPS, no 3, p. 275.

Amélioration d'image, no 4, p. 366.

Aménagement, no 3 , p. 259.

Aménagement de la néonymie, n 3 , p. 259.

Analyse, $n^{\circ} 3$, p. 200.

Analyse, effort d', $\mathrm{n}^{\circ} 1$, p. 44

Analyse, phase d', no 3, p. 203

Analyse, tache d', $n^{\circ} 4$, p. 377 .

Angle d'éclairage, $n^{\circ} 4$, p. 366.

Angle d'inclinaison, no 4 , p. 366 .

Angle d'irradiation, no 4, p. 366.

Angle de dépression, no 4 , p. 366 .

Angle de dépression à l'horizon, $n^{\circ} 4$, p. 366.

Angle de visée, $n^{\circ} 4$, p. 366.

Animal à croissance rapide, no 4, p. 361 .

Animal de bonne venue, no 4 , p. 361 .

Animal faisant bien, $n^{\circ} 4$, p. 361 .

Animal, par, no 4 , p. 361.

Antenne latérale, radar aéroporté, no 4 , p. 374.

Antenne synthétique, radar à, no 4, p. 374 .

Anticipation cognitive, no 4 , p. 353 .

Anticipation de langue, no 4 , p. 353

Anticipation du sens, no 4 , p. 353.

Anticipation, importance de, $\mathrm{n}^{\circ} 1$, p. 46

Anticipation intellectuelle, no 4, p. 353.

Anticipation linguistique, n० 4, p. 353.

Anticipation sémantique, no 4 , p. 354.

Anticipation sensique, $n^{\circ} 4$, p. 354 .

Anticipation verbale, no 4 , p. 354 .

Aplomb, d', no 4, p. 362 .

Appareil de détente, $n^{\circ} 3$, p. 267.
Appauvrissement lexical, no 3, p. 292.

Apport, avion d', no 4 , p. 382 .

Apport, transporteur d', no 4 , p. 381.

Appréhension du sens, $n^{\circ} 4$, p. 354.

Arborescence à progression horizontale, $n^{\circ} 2$, p. 163.

Arborescence à progression verticale, $n^{\circ} 2$, p. 163.

Arborescence horizontale, $\mathrm{n}^{\circ} 2$, p. 163.

Arborescence verticale, no 2 , p. 163.

Arbre analogique, $\mathrm{n}^{\circ} 2$, p. 161 .

Arbre de domaine, $n^{\circ} 2$, p. 161.

Arbre généalogique, $\mathrm{n}^{\circ} 2$, p. 161

Arburation interne, no 3, p. 272.

Arène, $n^{\circ} 4$, p. 362.

Assimilation du sens, $n^{\circ} 4$, p. 354 .

Assimilation graphique, $n^{\circ} 2$, p. 121

Assimilation phonique, $\mathrm{n}^{\circ} 2, \mathrm{p} .120$.

ATR, no 4, p. 382 .

Attache de la queue, $n^{\circ} 4$, p. 363 .

Audio-visuel connecté, no 4 , p. 337.

Auteur, droit d', no 4 , p. 400 .

Autoinflammation, $\mathrm{n}^{\circ} 3$, p. 271.

Avion court-courrier, $\mathrm{n}^{\circ} 4$, p. 382

Avion d'apport, $n^{\circ} 4$, p. 382 .

Avion de transport régional, no 4 , p. 382 .

Avion régional, $n^{\circ} 4$, p. 382 .

Baccalauréat en traduction, programme de, $n^{\circ} 4$, p. 405 .

Bagage cognitif, $n^{\circ} 1$, p. 22 ; no 3 , p. 207 ; no 4 p. 354 .

Balayage, radiomètre à, no 4 , p. 374 .

Bande, largeur de, $n^{\circ} 4$, p. 371 .

Base arborescente, $n^{\circ} 4$, p. 333.

Base de données multicritère, no 4 , p. 332.

Bâton, n० 4, p. 362.

Benjamin, no 1 , p. 10 .

Benjamin, Walter, no 1, p. 10.

Berman, Antoine, $n^{\circ} 4$, p. 341

Berne, convention de, n ${ }^{\circ} 4$, p. 400 .

Besoin linguistique, no 4 , p. 320

Besoin protéique, no 4 , p. 362 .

Besoins alimentaires, $n^{\circ} 4$, p. 361 .

Bétail de sélection, $n^{\circ} 4$, p. 360 .

Biunivocité, no 2, p. 125.

Bceuf, pis de, no 4 , p. 360 .

Bouf, viande de, no 4 , p. 359.

Boucherie, bovin de, no $^{\circ}$, p. $359 ; n^{\circ} 4$, p. 360

Bouquet, no 4, p. 363.

Bouton, Charles P., no 4, p. 395.

Bouvillon, no 4 , p. 360 .

Bovin de boucherie, no 4 , p. 359

Box, $n^{\circ} 4$, p. 362.

Brosse de paille de riz, $n^{\circ} 4$, p. 362 .

Cage de contention, $n^{\circ} 4$, p. 362 .

Calibrage, $n^{\circ} 4$, p. 366

Calibration, $n^{\circ} 4$, p. 366

Calque, n०3, p. 212.

Capteur, no 4 , p. 366 .

Capteur actif, n०4, p. 367.

Capteur de luminescence, no 4, p. 367. 
Capteur en peigne, $n^{\circ} 4$, p. 367.

Capteur passif, $n^{\circ} 4$, p. 367 .

Caractère descriptif, $n^{\circ} 2$, p. 124.

Carburation externe, $n^{\circ} 3$, p. 272.

Carburation préalable, $n^{\circ} 3$, p. 272.

Carcasse, classement de, no 4, p. 363.

Carte infographique, $n^{\circ} 4$, p. 367 .

Carte informatique, $n^{\circ} 4$, p. 367

Carte informatisée, $n^{\circ} 4$, p. 367.

Case, no 4 , p. 362 .

Cellule détectrice, $n^{\circ} 4$, p. 367

Cellule germinale, $n^{\circ} 4$, p. 361 .

Chaîne sonore, $\mathrm{n}^{\mathrm{0}} 1, \mathrm{p} .20$.

Chair, rendement en, $n^{\circ} 4$, p. 362.

Chaleur, pompe à, no 3 , p. 261.

Chambre multibande, $n^{0} 4$, p. 367.

Chambre photographique, $n^{\circ} 4$, p. 367 .

Champ conceptuel-fonctionnel, $n^{\circ} 4$, p. 333

Champ fonctionnel-conceptuel, $n^{\circ} 4$, p. 333.

Champ phraséologique, $n^{\circ} 4, p .333$.

Chanfrein, $n^{\circ} 4$, p. $360 ; n^{\circ} 4$, p. 362.

Charpente, équilibre de la, $n^{\circ} 4$, p. 363.

Charte des droits et libertés, no 4 , p. 405.

Chatoiement, $n^{\circ} 4$, p. 378.

Châtron, $n^{\circ} 4$, p. 360

Choix de l'émetteur, $n^{\circ} 2$, p. 139.

Choix des sélectionneurs, $n^{\circ} 4$, p. 360 .

Circuit d'allumage, $n^{\circ} 3$, p. 272.

Circuit d'injection, $n^{\circ} 3$, p. 273.

Clarté, no 2, p. 146.

Classe de qualité bouchère, $n^{\circ} 4$, p. 362 .

Classement de carcasse, no $n^{\circ}$, p. 363 .

Coefficient de foisonnement $n^{\circ} 2$, p. 145

Coffre d'exposition, $n^{\circ} 4$, p. 362.

Cohyponyme, no 2 , p. 121.

Collègue passif, $\mathrm{n}^{\circ} 3$, p. 203.

Collision homonymique, $\mathrm{n}^{\circ} 2$, p. 125.

Collision polysémique, $\mathrm{n}^{\circ} 2$, p. 125 .

Combustion diesel, $n^{\circ} 3$, p. 273.

Combustion vive, $\mathrm{n}^{\circ} 3$, p. 273.

Communication, efficacité de la, no 2, p. 119.

Communication opérationnelle, $n^{\circ} 4$, p. 320 .

Compatibilité lexicale, $n^{\circ} 2$, p. 121.

Compensation extra-linguistique, $\mathrm{n}^{\mathrm{0}} 4$, p. 321 .

Compétence linguistique, no 4 , p. 326 .

Complément cognitif, $n^{\circ} 4$, p. 354 .

Compléments pour jeunes animaux, $n^{\circ} 4$, p. 360 .

Composition colorée, $n^{\circ} 4$, p. 367.

Compréhension, $n^{\circ} 3$, p. 276.

Compréhension du discours, $n^{\circ} 1$, p. 32 .

Conceptualisation, $n^{\circ} 4$, p. 354.

Concision terminologique, $n^{\circ} 2$, p. 120

Concours, $n^{\circ} 4$, p. $359 ; n^{\circ} 4$, p. 362

Concours d'expertise, $n^{\circ} 4$, p. 361 .

Concours de jugement, $n^{\circ} 4$, p. 361.

Concours, licou de, no 4 , p. 362 .

Conditionnement, $n^{\circ} 4$, p. 361 .

Conditionnement du pelage, $n^{\circ} 4$, p. 361 .

Conditionnement du poil, $n^{\circ} 4$, p. 361 .

Conditionnement, période, no 4 , p. 360.

Conférence internationale, $n^{\circ} 3$, p. 199.

Conférence, interprétation, $n^{\circ} 4$, p. 320 .

Conférence, organisateur de, no 1, p. 103.

Conférence technique, no 3 , p. 199.

Conférences, langage des, $n^{\circ} 4$, p. 324.
Connaissance des langues, $n^{\circ} 4, p .320$

Connaissance partagée, $n^{\circ} 4$, p. 354

Connaissance spécialisée, no 3 , p. 207.

Connotation, $\mathrm{n}^{\circ} 4$, p. 354 .

Consanguinité, no 4 , p. $360 ; n^{\circ} 4$, p. 361

Console de visualisation, $n^{\circ} 4$, p. 367 .

Consultation automatique, $n^{\circ} 4$, p. 335

Contact des langues, $\mathrm{n}^{\circ} 2$, p. 122 .

Contention, cage de, $n^{\circ} 4$, p. 362 .

Contention, couloir de, no 4 , p. 360 .

Contenu cognitif, $\mathrm{n}^{\circ} 1$, p. 26.

Contexte cognitif, $n^{\circ} 4$, p. 355 .

Contexte verbal, $n^{\circ} 4$, p. 355.

Contrainte, couloir de, $n^{\circ} 4$, p. 360.

Contrainte linguistique, $n^{0} 2$, p. 139.

Convention de Berne, no 4, p. 400.

Convention internationale, $n^{\circ} 4$, p. 400 .

Convention universelle de Genève, no 4 , p. 400 .

Corde, $n^{\circ} 4$, p. 362.

Cornadis, $n^{\circ} 4$, p. 360

Couleur, affichage en, $n^{\circ} 4$, p. 365 .

Couleur composée, image en, no 4, p. 370.

Couloir de contention, $\mathrm{n}^{\circ} 4$, p. 360 .

Couloir de contrainte, $n^{\circ} 4$, p. 360.

Couloir exploré, $n^{\circ} 4$, p. 368 .

Couloir, largeur de, $n^{\circ} 4$, p. 371

Cours à l'adaptation, no 3 , p. 274.

Court-courrier, avion, no 4 , p. 382.

Court terme, mémoire à, $n^{0} 4$, p. 355 .

Coûts d'engraissement, $n^{\circ} 4$, p. 361 .

Crédibilité, maintien de la, n⿳⺈, p. 207.

Critère d'acceptabilité, $\mathrm{n}^{\circ} 2$, p. 120.

Critère de sélection, $\mathrm{n}^{\circ} 2$, p. 120 .

Critère de terminologie, $n^{\circ} 2$, p. 120 .

Critère linguistique, $\mathrm{n}^{\circ} 2$, p. 119 .

Croisement, $n^{\circ} 4$, p. 360 .

Croisement, élevage en, $n^{\circ} 4$, p. 360 .

Croisement en rotation, $n^{\circ} 4$, p. $360 ; n^{\circ} 4$, p. 362.

Croisement industriel, $n^{\circ} 4$, p. 360.

Croisement réciproque, no 4 , p. $360 ; n^{\circ} 4$, p. 362 .

Croisement terminal, $n^{\circ} 4$, p. $360 ; n^{\circ} 4$, p. 363 .

Cycle (à combustion) à pression constante, $\mathrm{n}^{\mathrm{O}} 3$, p. 266.

Cycle (à combustion) à volume constant, no 3 , p. 266.

Cycle de Beau de Rochas, no 3, p. 266.

Cycle de Joule tronqué, $\mathrm{n}^{\mathrm{o}} 3$, p. 266 .

Cycle théorique, $n^{\circ} 3$, p. 265 .

Cycle thermodynamique, $n^{\circ} 3$, p. 263.

De race, $n^{\circ} 4$, p. 360 .

Découpe, $n^{\circ} 4$, p. 360

Défaillance, $\mathrm{n}^{\circ} 1$, p. 45 .

Définition, $\mathrm{n}^{\circ} 2$, p. 125

Déflagration, $n^{\circ} 3$, p. 273.

Déflagration, moteur à, $\mathrm{n}^{\circ} 3$, p. 273.

Densité informationnelle, no 4 , p. 325

Déontologie, $n^{\circ} 1$, p. 78.

Dépression à l'horizon, angle de, no 4 , p. 366.

Dépression, angle de, no 4, p. 366.

Dérivabilité, no 2 , p. 122.

Derrida, J., no 1, p. 10.

Désambiguisation, $\mathrm{n}^{\circ} 1$, p. 22. 
Descripteur, no 4 , p. 332.

Détendu, $\mathrm{n}^{\circ} 3$, p. 263.

Détente, $\mathrm{n}^{\circ} 3$, p. 261.

Détente isotherme, $\mathrm{n}^{\circ} 3$, p. 263

Détonation, $\mathrm{n}^{\circ} 3$, p. 273 .

Détonations, moteur à, no 3 , p. 273.

Détour historique, $\mathrm{n}^{\circ} 1$, p. 11 .

Deuxième niveau, $\mathrm{n}^{\circ} 4$, p. 379.

Développement de la pensée, $\mathbf{n}^{\circ} 3$, p. 236

Déverbalisation, $\mathrm{n}^{\circ} 1$, p. 26

Déversement en radar, $n^{\circ} 4$, p. 368.

Déversement-radar, $\mathrm{n}^{\circ} 4$, p. 368.

Diagramme de Clapeyron, $\mathrm{n}^{\circ} 3$, p. 262.

Diagramme dynamique, $\mathrm{n}^{\circ} 3$, p. 262.

Diagramme P-V, no 3, p. 262.

Diapositive pilotée, $n^{\circ} 4$, p. 332.

Dictionnaire aérospatial, no 4 , p. 398.

Dictionnaire automatique, $n^{\circ} 4$, p. 332 .

Dictionnaire bilingue, $\mathrm{n}^{\mathrm{O}} 3$, p. 242.

Discours, compréhension du, $\mathrm{n}^{\circ} 1$, p. 32 .

Discours, exégèse du, no 3, p. 199.

Discours physique du langage, $n^{\circ} 4$, p. 395.

Discours, production du, $n^{\circ} 4$, p. 323 .

Discours scriptural oral, $n^{\circ} 4$, p. 324

Distance-temps, $n^{\circ} 4$, p. 368 .

Distance-temps au sol, $n^{\circ} 4$, p. 368 .

Distinctivité, $\mathrm{n}^{\circ} 2$, p. 121.

Documentation, $n^{\circ} 3$, p. 200

Domaine corrigée, $n^{\circ} 4$, p. 368 .

Domptage, $n^{\circ} 4$, p. 360.

Donnée auxiliaire, $\mathrm{n}^{\circ} 4$, p. 368 .

Donnée brute, $n^{\circ} 4$, p. 368.

Donnée de terrain, $n^{\circ} 4$, p. 369

Donnée élaborée, no 4 , p. 369

Donnée prétraitée, $n^{\circ} 4$, p. 369

Dos, ligne du, $\mathrm{n}^{\circ} 4$, p. 363.

Double langage, $n^{\circ} 1$, p. 19

Dressage, $n^{\circ} 4$, p. 361 .

Droit d'auteur, no 4 , p. 400

Droit québécois, no 4, p. 405 .

Échange sémiotique, $n^{\circ} 1$, p. 7

Échantillonnage, maille d', no 4 , p. 372.

Échantillonnage, pas d', $n^{\circ} 4$, p. 372.

Échelon lexical, $n^{\circ} 2$, p. 139.

Éclairage, angle d', no 4 , p. 366.

Écorner, no 4, p. 361.

Écoute, no 3, p. 200

Écoute, effort d', no 1, p. 44.

Écoute, phase d', no 3, p. 203

Écran, image d', no 4, p. 370 .

Effet de mer, $n^{\circ} 4$, p. 369.

Effet de rapprochement, $n^{\circ} 4$, p. 369

Effet de style, $\mathrm{n}^{\circ} 1$, p. 57

Efficacicé de la communication, $n^{\circ} 2$, p. 119.

Efficacité des termes, $n^{\circ} 2$, p. 120.

Effort d'analyse, $\mathrm{n}^{\circ} 1$, p. 44

Effort d'écoute, $\mathrm{n}^{\circ} 1$, p. 44.

Effort de mémoire, $\mathrm{n}^{\circ} 1$, p. 44.

Effort, intensité de $1^{\prime}, n^{\circ} 4$, p. 321 .

Effort, loi du moindre, $n^{0} 3$, p. 206.

Efforts, modèle d', no 1, p. 44 .

Élément perturbateur, nº 3, p. 201.
Élevage de sélection, $\mathrm{n}^{\circ} 4$, p. 360 .

Élevage en croisement, $n^{\circ} 4$, p. 360.

Elevage en lignées pures, $n^{\circ} 4$, p. $360 ; n^{\circ} 4$, p. 362.

Élevage en lignes droites, no 4 , p. 363.

Élevage, valeur d', $n^{\circ} 4$, p. 360 .

Élever, $n^{\circ} 4$, p. 360.

$\mathrm{EM}, \mathrm{n}^{\circ} 4$, p. 362.

Embouche, veau d', no 4, p. 361.

Embranchement, $n^{\circ} 2$, p. 167.

Émetteur, choix de l', $\mathrm{n}^{\circ} 2$, p. 139.

Empan de la mémoire immédiate, no 4, p. 355.

Empan mnésique, no 4, p. 355.

Enceinte de présentation, no 4 , p. 362.

Encodage, no 4, p. 329.

Énergie cinétique, $\mathrm{n}^{\circ} 3$, p. 261.

Énergie de pression, $n^{\circ} 3$, p. 261

Énergie mécanique, $n^{\circ} 3$, p. 261.

Énergie métabolisable, no 4, p. 362.

Engraissement, coûts d', $n^{\circ} 4$, p. 361.

Engraissement, parc d', no 4, p. 361 .

Engraissement, veau d', $\mathrm{n}^{\circ} 4$, p. 361 .

Énoncé, $n^{\circ} 2$, p. $139 ; n^{\circ} 4$, p. 350.

Énoncé informatif, $n^{\circ} 2$, p. 139.

Énonciation, mode d', no 3 , p. 207.

Enseignement de la traduction juridique, $n^{\circ} 4$, p. 407.

Enseignement des langues, $n^{\circ} 3$, p. 274.

Ensilage, $n^{\circ} 4$, p. 361 .

Épreuve, no 4, p. 369.

Epreuve minute, $\mathrm{n}^{\circ} 4$, p. 369.

Équidensité colorée, no 4 , p. 369.

Équilibre d'interprétation, no 1, p. $44 ; n^{\circ} 3$, p. 200 .

Équilibre de la charpente, $n^{\circ} 4$, p. 363.

Espace interprétatif, $\mathrm{n}^{\circ} 1$, p. 8 .

Espèce, no 4, p. 360

Esprit des lois, $n^{\circ} 4$, p. 405.

Essence, moteur à, no 3, p. 261.

Étalonnage, $n^{\circ} 4$, p. 369.

Etat théologique, $n^{\circ} 3$, p. 237.

Etats de la traduction, trois, $n^{\circ} 3$, p. 236.

Étrille, $n^{\circ} 4$, p. 362.

Étrille écossaise, $n^{\circ} 4$, p. 362

Exégèse du discours, $\mathrm{n}^{\circ} 3$, p. 199.

Exercice de paraphrase, no 4, p. 328.

Expérience, no 3 , p. 207

Expérience pédagogique, no 2, p. 139.

Expertise, concours d', no 4 , p. 361 .

Expertise technique, $\mathrm{n}^{\circ} 1$, p. 78.

Explication, no 3, p. 205.

Exploration horizontale, no 3, p. 201.

Explosion, moteur à, $\mathrm{n}^{\circ} 3$, p. $261 ; \mathrm{n}^{\circ} 3$, p. 273.

Exposant, qualités d', $n^{\circ} 4$, p. 362 .

Exposant, stick d', no 4, p. 362.

Exposant, talent d', $\mathrm{n}^{\circ} 4$, p. 362.

Exposition, no 4, p. $359 ; n^{\circ} 4$, p. 362.

Exposition, coffre d', $n^{\circ} 4$, p. 362 .

Exposition, licou d', $\mathrm{n}^{\circ} 4$, p. 362.

Expression, $n^{\circ} 1$, p. $65 ; n^{\circ} 3$, p. 276.

Expression familière, $\mathrm{n}^{\circ} 3$, p. 288.

Expression imagée, $\mathrm{n}^{\circ} 1$, p. 56.

Expression, maladresse d', no 3, p. 292. 
Fausse couleur, $\mathrm{n}^{\circ} 4$, p. 370 .

Faute de performance, $\mathrm{n}^{\circ} 3$, p. 292.

Faux-filet, $n^{\circ} 4$, p. 362

Feed-back, no 1, p. 63.

Fiche, no 4, p. 334.

Fidélité, $n^{\circ} 2$, p. 146

Fidélité d'une traduction, $n^{\circ} 4$, p. 355.

Filiation femelle, $n^{\circ} 4$, p. 361 .

Filiation mâle, $\mathrm{n}^{\circ} 4$, p. 362.

Finition, $n^{\circ} 4$, p. 363 .

Fluide moteur, $\mathrm{n}^{\circ} 3$, p. 261.

Foire agricole, no 4 , p. $359 ; n^{\circ} 4$, p. 362 .

Formation des traducteurs, no 2 , p. 139.

Formation spécialisée, $\mathrm{n}^{\circ} 1$, p. 78.

Foucault, no 3 , p. 236.

Fourrage, $n^{\circ} 4$, p. 361

Fourrage, utilisation du, $n^{\circ} 4$, p. 361 .

Frais d'alimentation, $n^{\circ} 4$, p. 361

Front de flamme, $n^{\circ} 3$, p. 273.

Gain informationnel, $\mathrm{n}^{\circ} 2$, p. 142.

Ganache, $n^{\circ} 4$, p. 361 ; no 4, p. 362 .

Genève, convention universelle de, $n^{\circ} 4$, p. 400 .

Génisse, $n^{\circ} 4$, p. 361.

Géniteur, $n^{\circ} 4$, p. 362.

Gestante, no 4 , p. 360 .

GETA, n' 3, p. 275.

Glose périphrastique, no 3 , p. 243.

Glossaire de la thếorie interprétative, $n^{\circ} 4$, p. 352 .

Grain de provende, $n^{\circ} 4$, p. 361.

Grandeur physique, $n^{\circ} 3$, p. 261

Graphocentrisme, $\mathrm{n}^{\mathbf{0}} 1$, p. 10.

Gras cardiaque, $n^{\circ} 4$, p. 361 .

Gras de noix de côte, $n^{\circ} 4$, p. 362.

Gras dorsal, no 4, p. 359

Gras pelvien, $n^{\circ} 4$, p. 361 .

Gras rếnal, $\mathrm{n}^{\circ} 4$, p. 361.

Gravide, $n^{\circ} 4$, p. 360

Grille conceptuelle, $\mathbf{n}^{\circ} 1$, p. 30.

Groupe-capteur, $n^{\circ} 4$, p. 370.

Groupe-témoin, $n^{\circ} 4$, p. 360.

Guide, $n^{\circ} 4$, p. 362.

HAMT, no 3 , p. 275.

Haut sur pattes, no 4 , p. 362.

Herd-book, no 4, p. 361 .

Histoire de la pensée, $n^{\circ} 3$, p. 236

Histoire de la traduction, $\mathrm{n}^{\circ} 4$, p. 405.

Homogénéité de la terminologie, $n^{\circ} 2$, p. 122

Hyperbole équilatère, $n^{\circ} 3$, p. 263.

Hyperfréquence, scanneur en, no 4 , p. 376.

Hyperonyme, $\mathrm{n}^{\circ} 2$, p. 121

Hyponyme, $\mathbf{n}^{\circ}$ 2, p. 121.

I.R.C., n $^{\circ} 4$, p. 371

Idéaliste, $\mathrm{n}^{\circ} 1, \mathrm{p} .12$.

Idée, $\mathrm{n}^{\circ} 2$, p. 139.

Idiotisme, no 3, p. 242.

Image, $n^{\circ} 4$, p. 370 .

Image, amélioration d', $n^{\circ} 4$, p. 366
Image composite, $n^{0} 4$, p. 370

Image composite couleurs, $n^{\circ} 4$, p. 370 .

Image d'écran, $n^{\circ} 4$, p. 370.

Image en couleur composée, $n^{\circ} 4$, p. 370 .

Image photographique, $n^{\circ} 4$, p. 370 .

Image potentielle, $n^{\circ} 4$, p. 370 .

Image, rémanence de, $n^{\circ} 1$, p. 57.

Image, résolution de $\mathrm{l}^{\prime}, \mathrm{n}^{\circ} 4$, p. 375 .

Image, restauration d', $n^{\circ} 4$, p. 375 .

Image-satellite, $n^{\circ} 4$, p. 370 .

Image transposable, $\mathrm{n}^{\circ} 1$, p. 58.

Image-vidéo, $n^{\circ} 4$, p. 371 .

Imagerie, $n^{\circ} 4$, p. 371 .

Images, superposition d', no 4, p. 377.

Imageur, $n^{\circ} 4$, p. 371.

Impensé du texte, $n^{\circ} 1$, p. 8.

Implicite linguistique, no 3 , p. 294.

Importance de l'anticipation, $\mathrm{n}^{\circ} 1$, p. 46.

Impropriété, $\mathrm{n}^{\mathrm{o}} 3$, p. 292.

Improvisation, $\mathrm{n}^{\circ} 1$, p. $56 ; \mathrm{n}^{\circ} 3$, p. 207.

Inclinaison, angle $d^{\prime}, n^{\circ} 4$, p. 366 .

Indice phonétique, $\mathrm{n}^{\circ} 1$, p. 62

Infographie, no 4 , p. 371.

Information sensorielle, $n^{\circ} 1$, p. 21

Informatique, $\mathrm{n}^{\circ} 3$, p. 274.

Infrarouge couleur, $n^{\circ} 4$, p. 371

Injection, $n^{\circ} 3$, p. 272.

Intégration, $\mathrm{n}^{\circ} \mathbf{2}$, p. 120

Intégrité du message, $\mathrm{n}^{\circ} 4$, p. 321

Intelligence artificielle, $n^{\circ} 3$, p. 236

Intensité de l'effort, $\mathrm{n}^{\circ} 4$, p. 321 .

Interrégional, transport aérien, $n^{\circ} 4$, p. 383.

Interférence, $\mathrm{n}^{\circ} 1$, p. 59.

Interférence linguistique, $\mathrm{n}^{\circ} 1$, p. $59 ; \mathrm{n}^{\circ} 3$, p. 201

Interférence, loi de la moindre, $\mathrm{n}^{\circ} 3$, p. 206.

Interlingual, $\mathrm{n}^{\circ} 1$, p. 10.

Interprétariat, $\mathrm{n}^{\circ} 1$, p. 19.

Interprétation, $\mathrm{n}^{\circ} 1$, p. $19 ; \mathrm{n}^{\circ} 1$, p. $25 ; \mathrm{n}^{\circ} 3$, p. 199.

Interprétation consécutive, $n^{\circ} 4$, p. 322 .

Interprétation de conférence, $n^{\circ} 1$, p. $19 ; n^{\circ} 4$, p. 320.

Interprétation, équilibre d', no 1, p. 44 ; no 3, p. 200.

Interprétation, processus de $1, \mathrm{n}^{\mathrm{o}} 1, \mathrm{p} .20$.

Interprétation simultanée, $\mathrm{n}^{\circ} 1$, p. $44 ; \mathrm{n}^{\circ} 4$, p. 322 ; $n^{\circ} 4$, p. 323.

Interprète de conférence, responsabilité de $l^{\prime}, \mathrm{n}^{\circ} 1$, p. 78.

Intersémiotique, $\mathrm{n}^{\circ} 1, \mathrm{p} .10$.

Intra-, $\mathrm{n}^{\circ} 1$, p. 10.

Inversion du principe de la forme, $n^{0} 4$, p. 327.

Irradiation, angle $d^{\prime}, n^{\circ} 4$, p. 366 .

Isobare, $\mathrm{n}^{\circ} 3, \mathrm{p} .263$.

Jakobson, $\mathrm{n}^{\circ} 1$, p. 9.

Jeu des motivations, $n^{\circ} 3$, p. 242.

Jugement, concours de, no 4 , p. 361.

Juger, $n^{\circ} 4$, p. 361

Lacune informationnelle, $\mathrm{n}^{\circ} 2$, p. 145 .

Laisse, $n^{\circ} 4$, p. 362 .

Langage artificiel, $\mathrm{n}^{\circ} 2$, p. 163.

Langage de la procédure, $n^{\circ} 4$, p. 324 . 
Langage des assemblées, n० 1, p. 65 .

Langage des conférences, $n^{\circ} 4$, p. 324

Langage non spécialisé, no 4 , p. 324.

Langage oratoire, $n^{\circ} 4$, p. 324 ; n 4 , p. 325.

Langage technique, $n^{\circ} 4$, p. 325 .

Langue active, no 4 , p. $320 ; n^{\circ} 4$, p. 323.

Langue, anticipation de, $n^{\circ} 4$, p. 353 .

Langue de spécialité, $\mathrm{n}^{\circ} 2$, p. $119 ; \mathrm{n}^{\circ} 3$, p. 257.

Langue française, promotion de la, no 4, p. 342.

Langue française, traduction et, $n^{\circ} 4$, p. 341.

Langue passive, $n^{\circ} 1$, p. 59 ; n० 4 , p. $320 ; n^{\circ} 4$, p. 323 .

Langue, sens de la, no 1 , p. 59.

Langues, connaissance, no 4 , p. 320 .

Langues, enseignement des, $n^{\circ} 3$, p. 274.

Largeur de bande, $n^{\circ} 4$, p. 371.

Largeur de couloir, no 4 , p. 371 .

Lasso, no 4 , p. 361.

Le médecin malgré lui, no 4 , p. 316

Lecture, n०3, p. 207.

Législation belge, $\mathrm{n}^{\circ} 4$, p. 401 .

Lexique, limite du, no 3, p. 242.

Licol, $\mathrm{n}^{\circ} 4$, p. 361.

Licou, no 4 , p. 361 .

Licou d'exposition, no 4 , p. 362

Licou de concours, no 4 , p. 362

Ligne du dos, $n^{\circ} 4$, p. 363

Lignée généalogique, no 4 , p. 361 .

Lignée maternelle, no 4 , p. 361 .

Lignée paternelle, no 4 , p. 362.

Lignées pures, élevage en, $\mathrm{n}^{\circ} 4$, p. $360 ; \mathrm{n}^{\circ} 4$, p. 362 .

Lignes droites, élevage en, no 4, p. 363.

Limite de résolution, $n^{\circ} 4$, p. 371 .

Limite de résolution au sol, no 4, p. 371.

Limite de résolution radiométrique, no 4 , p. 371.

Limite de résolution spatiale, no 4 , p. 371 .

Limite de résolution spectrale, n०4, p. 372.

Limite de séparation spatiale, $n^{\circ} 4$, p. 372.

Limite du lexique, no 3, p. 242.

Lisibilité, no 2, p. 125.

Littéralisme, no 3 , p. 238.

Livre généalogique, no 4 , p. 361 .

Locution, no 3 , p. 242

LOGOS, n' 3, p. 275.

Loi de la moindre interférence, $n^{\circ} 3$, p. 206

Loi du moindre effort, no 3 , p. 206.

Lois, esprit des, n⿳4, p. 405.

Long terme, mémoire à, no 4 , p. 355.

Longe, n०4, p. 361 ; n०4, p. 362 .

Luminescence, capteur de, $n^{\circ} 4$, p. 367 .

Machine thermique, $n^{\circ} 3$, p. 261.

MAHT, no 3 , p. 275 .

Maille d'échantillonnage, $n^{\circ} 4$, p. 372.

Maintien de la crédibilité, n० 3 , p. 207.

Maladresse d'expression, $\mathrm{n}^{\circ} 3$, p. 292.

Manière de traduire, no 3 , p. 236

Marché professionnel, $n^{\circ} 3$, p. 276.

Mégacalorie, $n^{\circ} 4$, p. 362 .

Mémoire à court terme, no 4, p. 355

Mémoire à long terme, no 4, p. 355 .

Mémoire auditive, no 4 , p. 355 .

Mémoire cognitive, no 4 , p. 355 .
Mémoire conceptuelle, n 4, p. 356.

Mémoire différée, $n^{\circ} 4$, p. 356.

Mémoire, effort de, $n^{\circ} 1$, p. 44.

Mémoire immédiate, no 4 , p. 356.

Mémoire immédiate, empan de la, no 4, p. 355.

Mémoire opérationnelle, $n^{\circ} 4$, p. 356

Mémoire verbale, $n^{\circ} 4$, p. 356 .

Mer, effet de, no 4 , p. 369.

Mére, $\mathrm{n}^{\circ} 4$, p. 361 .

Meschonnic, Henri, no 1 , p. 10.

Message, $n^{\circ} 2$, p. $119 ; n^{\circ} 4$, p. 350 .

Message, intégrité du, no 4 , p. 321 .

Métaphore, $n^{\circ} 1$, p. 56.

Métaphore terminologique, $\mathrm{n}^{\circ} 2, \mathrm{p} .124$.

Méthode de travail, no 3, p. 199.

Métier d'interprète, $n^{\circ} 1$, p. 101.

Micro-banque de terminologie, $n^{\circ} 4$, p. 332.

Micro-encyclopédie, no 4 , p. 332 .

Micro-informatique, $n^{\circ} 4$, p. 332 .

Microdictionnaire, $\mathrm{n}^{\circ} 4$, p. 332 .

Mise-bas, n4, p. 360.

Mode d'énonciation, n० 3, p. 207.

Modèle d'efforts, no 1, p. 44.

Moindre effort, loi du, no 3, p. 206.

Moment d'abattage, no 4, p. 362.

Monosémie, no 2, p. 125.

Monovalence, $\mathrm{n}^{\circ} 2$, p. 125.

Montréal, Université de, no 4, p. 405.

Morceau primaire, $n^{\circ} 4$, p. 360 .

Morceau secondaire, $n^{\circ} 4$, p. $360 ; n^{\circ} 4$, p. 362

Morphème-mot, no 3 , p. 242.

Mot terminogène, $n^{\circ} 2$, p. 123

Mot traduisible, no 4 , p. 356.

Mot transcodable, no 4, p. 356.

Moteur à allumage commandé, no 3, p. 272.

Moteur à allumage par compression, $\mathrm{n}^{\circ} 3$, p. 271.

Moteur à allumage par étincelle, no 3, p. 272 .

Moteur à combustion interne, no 3 , p. 268.

Moteur à déflagration, $n^{\circ} 3$, p. 273.

Moteur à détonations, no 3, p. 273.

Moteur à essence, $n^{\circ} 3$, p. 261.

Moteur à explosion, $n^{\circ} 3$, p. $261 ; n^{\circ} 3$, p. 273.

Moteur cinétique, n०3, p. 267.

Moteur de Stirling, n० 3 , p. 267.

Moteur de type volumétrique, no 3, p. 267.

Moteur diesel, n 3, p. 260.

Moteur électrique, $n^{\circ} 3$, p. 261.

Moteur hydraulique, no 3, p. 261.

Moteur proprement dit, no 3 , p. 268.

Moteur thermique, $n^{\circ} 3$, p. 261.

Motif, no 2, p. 123.

Motif, place du, no 2 , p. 123.

Motivation, $\mathrm{n}^{\circ} 2$, p. 123.

Motivations, jeu des, no 3, p. 242.

Naisseur, no 4, p. 360.

Naturalisation, n० 3 , p. 205.

Naturalisation sauvage, $n^{\circ} 3$, p. 205.

Néonyme, $n^{\circ} 2$, p. 120.

Néonymie, no 3 , p. 257

Néonymie, aménagement de la, no 3, p. 259.

Neurolinguistique, $n^{\circ} 4$, p. 395.

Niveau de traduction, $\mathrm{n}^{\circ} 1$, p. 27. 
Noix de côte, gras de, $\mathrm{n}^{\circ} 4$, p. 362.

Non-dit, $\mathrm{n}^{\circ} 1$, p. 8.

Normalisation, $n^{\circ} 3$, p. 257.

Nuage d'huile pulvérisée, $n^{\circ} 4$, p. 362 .

Omission, no 3, p. 204.

Onglon, $n^{\circ} 4$, p. 361 .

Opération textuelle, no 1 , p. 9.

Opération traduisante, no 1, p. $7 ; n^{\circ} 3$, p. 236.

Opératrice de changement, $\mathrm{n}^{\circ} 1, \mathrm{p} .7$.

Oralisation des connaissances, $n^{\circ} 4$, p. 328 .

Oralité, $\mathrm{n}^{\circ} 1$, p. 30.

Orateur, vitesse de l', no 3, p. 207.

Organigramme, $\mathrm{n}^{\circ} 2$, p. 161 .

Organisateur de conférence, $\mathrm{n}^{\circ} \mathrm{l}, \mathrm{p} .103$.

Ottawa, Université d', no 4, p. 405.

Ouverture synthétique, radar à, no 4, p. 374.

Paille de riz, brosse de, $n^{\circ} 4$, p. 362.

Pansage, $n^{\circ} 4$, p. 361.

Parage des sabots, $n^{\circ} 4$, p. 361 .

Paramètre de la qualité, no 3 , p. 207.

Paraphrase, $n^{\circ} 3$, p. 205.

Paraphrase, exercice de, $n^{\circ} 4$, p. 328.

Parc d'engraissement, $n^{\circ} 4$, p. 361.

Paronyme, $\mathrm{n}^{\circ} 2$, p. 123.

Pas d'échantillonnage, $n^{\circ} 4$, p. 372

Passager des lignes complémentaires, no 4 , p. 382.

Passager des lignes d'apport, $n^{\circ} 4$, p. 382.

Passager des lignes régionales, $n^{\circ} 4$, p. 382

Paz, O., no 1, p. 9

Pédagogie de la traduction, $\mathrm{n}^{\circ} 3$, p. 293

Peigne, capteur en, $n^{\circ} 4$, p. 367.

Peirce, Ch. S., no 1 , p. 9.

Pelage, conditionnement du, $n^{\circ} 4$, p. 361 .

Pensée, développement, no 3 , p. 236

Pensée, histoire de la, $\mathrm{n}^{\circ} 3$, p. 236.

Père, no 4 , p. 362.

Perfectionnement linguistique, $n^{\circ} 4$, p. $325 ; n^{\circ} 4$, p. 327.

Performance, faute de, $n^{\circ} 3$, p. 292.

Période de conditionnement, $n^{\circ} 4$, p. 360 .

Période de repos, $n^{\circ} 4$, p. 321 .

Persillage, $n^{\circ} 4$, p. 362 .

Perte, no 3, p. 204.

Perte informationnelle, no 3 , p. 200.

Petit Larousse illustré 1985, no 3 , p. 282.

Petit-porteur, $n^{\circ} 4$, p. 382

Phase d'analyse, no 3 , p. 203.

Phase d'écoute, $n^{\circ} 3$, p. 203

Phase intermédiaire, $\mathrm{n}^{\circ} 1$, p. 26.

Phonocentrisme, $\mathrm{n}^{\circ} 1$, p. $7 ; \mathrm{n}^{\circ} 1$, p. 11.

Photo aérienne, $\mathrm{n}^{\circ} 4$, p. 372.

Photo-satellite, $n^{\circ} 4$, p. 372.

Photographie multibande, $n^{0} 4$, p. 372.

Photographie spatiale, $n^{\circ} 4$, p. 372 .

Pis de bouf, no 4 , p. 360 .

Pixel, $n^{\circ} 4$, p. 372.

Place de la traduction, $n^{\circ} 3$, p. 236.

Place du motif, $n^{\circ} 2$, p. 123

Plate-forme, $\mathrm{n}^{\circ} 4$, p. 373.

Pleine, $n^{\circ} 4$, p. 360 .
Poétologue, $\mathrm{n}^{\circ} 1$, p. 7.

Poids utile, $n^{\circ} 4$, p. 362 .

Poids vif, no 4, p. 361.

Poil, conditionnement du, $n^{\circ} 4$, p. 361 .

Politique de la terminologie, $n^{\circ} 4$, p. 342

Politique de la traduction, $n^{\circ} 4$, p. 342.

Politique linguistique du Québec, $n^{\circ} 3$, p. 288.

Polysémie des signifiants, $\mathrm{n}^{\circ} 1$, p. 23.

Polytropique, no 3 , p. 263.

Pompe à chaleur, $n^{\circ} 3$, p. 261.

Portée distale, $n^{\circ} 4$, p. 373.

Portée proximale, $n^{\circ} 4$, p. 373.

Pose, prendre la, $n^{\circ} 4$, p. 362 .

Poser, no 4 , p. 362.

Pouvoir de résolution, $n^{\circ} 4$, p. 373.

Pouvoir de résolution radiométrique, $\mathrm{n}^{\circ} 4$, p. 373.

Pouvoir de résolution spatiale, $\mathrm{n}^{\circ} 4$, p. 373.

Pouvoir de résolution spectrale, $n^{\circ} 4$, p. 373.

Pouvoir séparateur, $n^{\circ} 4$, p. 373.

Pratique discursive, $\mathrm{n}^{\circ} 1$, p. 11.

Premier choix, qualité de, $n^{\circ} 4$, p. 360 .

Prendre la pose, $n^{\circ} 4$, p. 362.

Préparateur, $n^{\circ} 4$, p. 361.

Préparation, $n^{\circ} 4$, p. 361.

Préparation cognitive, $n^{\circ} 3$, p. 208.

Préparation, stratégie de, no 3, p. $201 ; n^{\circ} 3$, p. 208.

Préparation terminologique, no 3, p. 208.

Préparer, $n^{\circ} 4$, p. 361.

Préposition, no 3, p. 274.

Présence mnésique, $n^{\circ} 4$, p. 356.

Présentation, enceinte de, $n^{\circ} 4$, p. 362.

Présentation, stick de, $n^{\circ} 4$, p. 362.

Pression, énergie de, $\mathrm{n}^{\circ} 3$, p. 261.

Principe de la forme, inversion du, $n^{\circ} 4$, p. 327.

Principe terminologique, $n^{\circ} 2$, p. 120.

Procédure, langage de la, $n^{\circ} 4$, p. 325 .

Processus de l'interprétation, no 1 , p. 20.

Processus de la traduction, $\mathrm{n}^{\circ} 1$, p. 20.

Processus mental, ${ }^{\circ} 1$, p. 59.

Production du discours, $n^{\circ} 4$, p. 323.

Production, effort de, $\mathrm{n}^{\circ} 1$, p. 44.

Production sémiotique, $\mathrm{n}^{\circ} 1, \mathrm{p} .7$.

Productivité, $n^{\circ} 2$, p. 122.

Programmathèque, $n^{\circ} 4$, p. 373.

Programme de baccalauréat en traduction, $n^{\circ} 4$, p. 405 .

Promotion de la langue française, $n^{\circ} 4$, p. 342.

Provende, grain de, $n^{\circ} 4$, p. 361.

Pulsation de la traduction, $n^{\circ} 1$, p. 7.

Pulsation opératoire, $\mathrm{n}^{\circ} 1$, p. 7 .

Qualité bouchère, classe de, no 4, p. 362 .

Qualité de premier choix, $n^{\circ} 4$, p. 360.

Qualité linguistique, $\mathrm{n}^{\circ} 2$, p. 146.

Qualité, paramètre de la, no 3 , p. 207.

Qualités d'exposant, $n^{\circ} 4$, p. 362 .

Qualités gustatives, $\mathrm{n}^{\circ} 4$, p. 362 .

Québec, politique linguistique du, no 3, p. 288.

Queue, attache de la, no 4 , p. 363.

Queue, toupillon de la, no 4, p. 363. 
R.A.A.S., no 4, p. 373

R.S.O., $n^{\circ} 4$, p. 375 .

R.V.L., $\mathrm{n}^{\circ} 4$, p. 375.

Rabattement-radar, $n^{\circ} 4$, p. 374

Race, no 4, p. 360.

Race (de), no 4, p. 362

Race, de, no 4, p. 363.

Race pure, no 4 , p. 362.

Rachat, valeur de, $\mathrm{n}^{\circ} 4$, p. 362 .

Radar à antenne synthétique, $n^{\circ} 4$, p. 374.

Radar à ouverture synthétique, $n^{\circ} 4$, p. 374 .

Radar à synthèse d'ouverture, no 4, p. 374.

Radar à visée latérale, $n^{\circ} 4$, p. 374.

Radar aéroporté à antenne latérale, no 4 , p. 374.

Radar, déversement en, $n^{\circ} 4$, p. 368.

Radar imageur, $n^{\circ} 4$, p. 374.

Radiomètre à balayage, $n^{\circ} 4$, p. 374 .

Radiomètre imageur, $n^{\circ} 4$, p. 374.

Rapprochement, effet de, $n^{\circ} 4$, p. 369.

Réalité de terrain, $\mathrm{n}^{\circ} 4$, p. 374.

Recommandation, $\mathrm{n}^{\circ} 3$, p. 259.

Rectiligne, $n^{\circ} 4$, p. 363.

Récupération de mots, $\mathrm{n}^{\circ} 1$, p. 62.

Rédaction technique, $n^{\circ} 3$, p. 286.

Rédaction, technique de la, $\mathrm{n}^{\circ} 3$, p. 287.

Réexpression, $\mathrm{n}^{\circ} 1, \mathrm{p} .56$.

Référence, troupeau de, $n^{\circ} 4$, p. 360 .

Réfiexe linguistique, $n^{\circ} 4$, p. 322 .

Réflexion théorique, $n^{\circ} 3$, p. 292.

Régional, transport aérien, $\mathrm{n}^{\circ} 4$, p. 383.

Registre inapproprié, $\mathrm{n}^{\circ} 1$, p. 56.

Registre linguistique, $n^{\circ} 4$, p. 324

Registre littéraire, $\mathrm{n}^{\circ} 1$, p. 55 .

Registre moyen, $n^{\circ} 1$, p. 55.

Registre original, $\mathrm{n}^{\circ} 1$, p. 55.

Règle pratique, $\mathrm{n}^{\circ} 1$, p. 7.

Réglementation belge, $n^{\circ} 4$, p. 401.

Relation générique, $\mathrm{n}^{\circ} 2$, p. 165

Relation partitive, $\mathrm{n}^{\circ} 2$, p. 165 .

Relief verbal, $n^{\circ} 1$, p. 61 .

Rémanence de l'image, $n^{\circ} 1$, p. 57.

Remplacement, vache de, $n^{\circ} 4$, p. 362.

Rendement, $n^{\circ} 4$, p. 361 .

Rendement à l'abattage, $n^{\circ} 4$, p. $360 ; n^{\circ} 4$, p. 361 .

Rendement de Carnot, n० 3, p. 265.

Rendement en chair, $n^{\circ} 4$, p. 362 .

Rendement théorique maximal, $n^{\circ} 3$, p. 265

Rendement thermodynamique théorique, $n^{\circ} 3$, p. 265.

Repos, période de, $\mathrm{n}^{\circ} 4$, p. 321.

Reproductibilité, $\mathrm{n}^{\circ} 2$, p. 122

Reproduction, $\mathrm{n}^{\circ} 3$, p. 205.

Résolution, no 4, p. 375.

Résolution au sol, limite de, no 4, p. 371.

Résolution de l'image, $\mathrm{n}^{\circ} 4$, p. 375 .

Résolution, limite de, $n^{\circ} 4$, p. 371.

Résolution, pouvoir de, $n^{\circ} 4$, p. 373

Résolution radiométrique, limite de, no 4, p. 371.

Résolution radiométrique, pouvoir de, no 4, p. 373.

Résolution spatiale, limite de, $n^{\circ} 4$, p. 371 .

Résolution spatiale, pouvoir de, $n^{\circ} 4$, p. 373.

Résolution spectrale, $n^{\circ} 4$, p. 375.

Résolution spectrale, limite de, $n^{\circ} 4$, p. 372.

Résolution spectrale, pouvoir de, no 4, p. 373.
Responsabilité de l'interprète de conférence, $\mathrm{n}^{\circ} 1$, p. 78.

Restauration d'image, $n^{\circ} 4$, p. 375.

Restitution, $n^{\circ} 4$, p. 323.

Retouche, no 4 , p. 363.

Réversible, no 3, p. 265.

Révolution radiométrique, $\mathrm{n}^{\circ} 4$, p. 375 .

Révolution spatiale, $n^{\circ} 4$, p. 375.

Rognage, $n^{\circ} 4$, p. 361.

Rotation, croisement en, $n^{\circ} 4$, p. $360 ; n^{\circ} 4$, p. 362.

S.M.B., $n^{\circ} 4$, p. 376

Sabot, $\mathrm{n}^{\circ} 4$, p. 361 .

Sabots, parage des, $n^{\circ} 4$, p. 361

Sacralisation, $n^{\circ} 1$, p. 10.

Sacralisation de l'écriture, $n^{\circ} 1$, p. 10.

Saillie, taxe de, $n^{\circ} 4$, p. 362.

Saint Jérôme, $n^{\circ} 3$, p. 237.

Salzburger Linguistische Analysen, no 4, p. 397

Sapidité, $\mathrm{n}^{\circ} 4$, p. 362.

Satellite SPOT, $n^{\circ} 4$, p. 364

Savoir fonctionnel, $n^{\circ} 4$, p. 336 .

Savoir organisé, $n^{\circ} 4$, p. 336.

Savoir partagé, $n^{\circ} 4$, p. 356.

Savoir ponctuel, $n^{\circ} 4$, p. 336.

Savoir, structuration du, $n^{\circ} 4$, p. 333.

Scannage, $n^{\circ} 4$, p. 375.

Scanner, $n^{\circ} 4$, p. 375 .

Scanneur en hyperfréquence, no 4, p. 375.

Scanneur multibande, $n^{\circ} 4$, p. 376.

Scanneur multibande modulaire, $\mathrm{n}^{\circ} 4$, p. 376.

Scanneuriste, $n^{\circ} 4$, p. 376.

Scannogramme, $n^{\circ} 4$, p. 376

Scannographe, $n^{\circ} 4$, p. 376.

Scannoscope, no 4, p. 376.

Scène, $n^{\circ} 4$, p. 376 .

Schleiermacher, $\mathrm{n}^{\circ} 1$, p. 12

Sélection, $n^{\circ} 4$, p. 360 .

Sélection, bétail de, $\mathrm{n}^{\circ} 4$, p. 360 .

Sélection, critère de, $\mathrm{n}^{\circ} 2$, p. 120

Sélection des tactiques, no 3, p. 206

Sélection, élevage de, $n^{\circ} 4$, p. 360 .

Sélection généalogique, $n^{\circ} 4$, p. 362 .

Sélectionné, $n^{\circ} 4$, p. 360 .

Sélectionneurs, choix des, no 4 , p. 360 .

Seleskovitch, $n^{\circ} 4$, p. 350

Sémantique, $\mathrm{n}^{\circ} 3$, p. 294.

Sémiose peircienne, $\mathrm{n}^{\circ} 1$, p. 10

Sens, $n^{\circ} 4$, p. 350.

Sens, anticipation du, $n^{\circ} 4$, p. 353.

Sens, appréhension du, $n^{\circ} 4$, p. 354 .

Sens, assimilation du, $n^{\circ} 4$, p. 354 .

Sens (d'un énoncé), $n^{\circ} 4$, p. 356.

Sens (d'un mot), no 4 , p. 357.

Sens de la langue, $n^{\circ} 1$, p. 59.

Sens pragmatique, $n^{\circ} 4$, p. 405 .

Sens pragmatique (d'un mot), $n^{\circ} 4$, p. 357.

Sens premier (d'un mot), $n^{\circ} 4$, p. 357.

Sensation, $n^{\circ} 1$, p. 21

Séparation spatiale, limite de, $\mathrm{n}^{\circ} 4$, p. 372.

Série lexicalisée, $n^{\circ} 3$, p. 242.

Serre, $n^{\circ} 4$, p. 360 .

Servitude linguistique, $n^{\circ} 2$, p. 142. 
Signature spectrale, $n^{\circ} 4$, p. 376

Signifiant, $n^{\circ} 1$, p. 23.

Signification, $n^{\circ} 4$, p. 349

Signification contextuelle (d'un mot), n ${ }^{\circ} 4$, p. 357.

Signification (d'un mot), no 4 , p. 357 .

Signification pragmatique (d'un mot), $n^{\circ} 4$, p. 357 .

Signifié systémo-sémantique, $\mathrm{n}^{\circ} 1$, p. 30

Situation, $n^{\circ} 4$, p. 350 .

Situation (interprétation), $\mathrm{n}^{\circ} 4$, p. 357

Sol, distance-temps au, $n^{\circ} 4$, p. 368

Spécialité, langue de, $\mathrm{n}^{\circ} 3$, p. 257.

Spontanéité, $\mathrm{n}^{\circ} 4$, p. 323 .

SPOT, satellite, $\mathrm{n}^{\circ} 4$, p. 364 .

Stabilisatrice du sens, $n^{\circ} 1$, p. 7.

Stabilité, $\mathrm{n}^{\circ} 2$, p. 126.

Stalle, $n^{\circ} 4$, p. 362 .

Stick d'exposant, $n^{\circ} 4$, p. 362

Stick de présentation, $n^{\circ} 4$, p. 362 .

Strate sémantique, no 1 , p. 22.

Stratégie de préparation, $\mathrm{n}^{\circ} 3$, p. $201 ; \mathrm{n}^{\circ} 3$, p. 208

Structuration des savoirs, $n^{\circ} 4$, p. 333

Structure arborescente, $n^{\circ} 2$, p. 161 .

Style, effet de, no 1, p. 57

Succulence, $n^{\circ} 4$, p. 362

Superposition d'images, $n^{\circ} 4$, p. 377.

Sur pied, $n^{\circ} 4$, p. 361

SUSY, $n^{\circ} 3$, p. 275.

Syntagme-fleuve, $\mathrm{n}^{\circ} 2$, p. 120

Synthèse d'ouverture, radar à, no 4, p. 374 .

Système terminologique-encyclopédique, $n^{\circ} 4$, p. 338.

Systémicité du terme, $\mathrm{n}^{\circ} 2$, p. 121

SYSTRAN, $n^{\circ} 3$, p. 275.

T.D.E.M., $n^{\circ} 4$, p. 377

Tache d'analyse, $n^{\circ} 4$, p. 377

Tache élémentaire, no 4, p. 377.

Tachèle, $n^{\circ} 4$, p. 377

Tactique, $\mathrm{n}^{\circ} 3$, p. 203

Tactiques, sélection des, $n^{\circ} 3$, p. 206.

Taille, $\mathrm{n}^{\circ} 4$, p. 361 .

Talent d'exposant, $n^{\circ} 4$, p. 362.

Taux de vêlage, $n^{\circ} 4$, p. 360 .

Tavelure, no 4, p. 379

Taxe de saillie, $n^{\circ} 4$, p. 362 .

Technique de la rédaction, no 3, p. 286

Télédétecteur, $\mathrm{n}^{\circ} 4$, p. 377 .

Télédétection, $n^{\circ} 4$, p. 377

Télédétection aérienne, $n^{\circ} 4$, p. 377.

Télédétection aérospatiale, $n^{\circ} 4$, p. $364 ; n^{\circ} 4$, p. 377.

Télédétection électromagnétique, $n^{\circ} 4$, p. 378 .

Télédétection spatiale, $n^{\circ} 4$, p. 378 .

Télédétectrice, $n^{\circ} 4$, p. 377 .

Téléinterprétation, $\mathrm{n}^{\circ} 4$, p. 378.

Terme, efficacité du, $n^{\circ} 2$, p. 120

Terminologie, $n^{\circ} 2$, p. 119 .

Terminologie, microbanque de, $n^{\circ} 4$, p. 332

Terminologie, politique de $1 a, n^{\circ} 4$, p. 342 .

Terrain, donnée de, $n^{\circ} 4$, p. 369 .

Terrain réalité de, no 4, p. 374.

Terrain, vérité de, $n^{\circ} 4$, p. 378

Test de validation, $\mathrm{n}^{\mathrm{o}} 2$, p. 167.
Texte canonique, $\mathbf{n}^{\circ} 1$, p. 9

Théoricien de la traduction, $n^{\circ} 3$, p. 239.

Théorie de la traduction, $n^{\circ} 1$, p. $10 ; n^{\circ} 4$, p. 405.

Théorie interprétative, glossaire de la, $n^{0} 4$, p. 353 .

Théorisation, $n^{\circ} 3$, p. 293

Thésaurus, $\mathrm{n}^{\circ} 2$, p. $161 ; \mathrm{n}^{\circ} 4$, p. 332 .

TITUS, $n^{\circ} 3$, p. 275 .

Toilettage, $n^{\circ} 4$, p. 361

Toilette, $\mathrm{n}^{\circ} 4$, p. 361 .

Tondage, $n^{\circ} 4$, p. 360

Tondeur, $n^{\circ} 4$, p. 360 .

Tondeuse, $n^{\circ} 4$, p. 360

Tondre, $n^{\circ} 4$, p. 360 .

Toupillon de la queue, $n^{\circ} 4$, p. 363 .

Traducteurs, formation des, $\mathrm{n}^{\circ} 2$, p. 139.

Traduction, $n^{\circ} 3$, p. $274 ; n^{\circ} 4$, p. 357.

Traduction assistée, $n^{\circ} 3$, p. 275.

Traduction automatique, $\mathrm{n}^{\circ} 3$, p. 275.

Traduction et langue française, $n^{\circ} 4$, p. 341 .

Traduction, fidélité d'une, $n^{\circ} 4$, p. 355 .

Traduction, histoire de la, $n^{\circ} 4$, p. 405.

Traduction juridique, enseignement de la, $n^{\circ} 4$ p. 407.

Traduction linguistique, $n^{\circ} 4$, p. 357

Traduction mythique, $\mathrm{n}^{\circ} 1$, p. 14.

Traduction, niveau de, $\mathrm{n}^{\circ} 1$, p. 27

Traduction orale, $\mathrm{r}^{\circ} 1$, p. 30

Traduction, pédagogie de la, $\mathrm{n}^{\circ} 3$, p. 293.

Traduction, politique de la, $n^{\circ} 4$, p. 342 .

Traduction, processus de la, no 1, p. 20.

Traduction-production, $\mathrm{n}^{\circ} 1$, p. 14

Traduction, programme de baccalauréat en, $n^{\circ} 4$, p. 405.

Traduction, pulsation de $1 \mathrm{a}, \mathrm{n}^{\circ} 1$, p. 7

Traduction, théoricien de la, no 3, p. 239

Traduction, théorie de la, $n^{\circ} 4$, p. 405

Traduction-transformation, $\mathrm{n}^{\circ} 1, \mathrm{p} .14$

Traduction, trois états de la, no 3, p. 236.

Traductologue, $\mathrm{n}^{\circ} 1$, p. 9 .

Traduire, manière de, $\mathrm{n}^{\circ} 3$, p. 236

Transcodage, $n^{\circ} 1$, p. 27 ; no 3 , p. $205 ; n^{\circ} 4$, p. 358.

Transfert d'idées, $\mathrm{n}^{\circ} 1$, p. 27 .

Transformation réelle, $\mathrm{n}^{\circ} 3$, p. 265

Transformation thermodynamique, $n^{\circ} 3$, p. 261

Transmission informationnelle, $\mathrm{n}^{\mathrm{O}} 4$, p. 323 .

Transphonation, $\mathrm{n}^{\circ} 1, \mathrm{p} .27$.

Transport aérien interrégional, $n^{\circ} 4$, p. 383

Transport aérien régional, $\mathrm{n}^{\circ} 4$, p. 383 .

Transport régional, avion de, n⿳⺈, p. 382 .

Transporteur aérien complémentaire, $n^{0} 4$, p. 381 .

Transporteur aérien régional, $n^{\circ} 4$, p. 381

Transporteur court-courrier, $n^{\circ} 4$, p. 381.

Transporteur d'apport, $n^{\circ} 4$, p. 381 .

Transporteur de troisième niveau, no 4 , p. 379 ; $n^{\circ} 4$, p. 381.

Transposition, $\mathrm{n}^{\circ} 4$, p. 358

Trièdre épistémologique, $\mathrm{n}^{\circ} 3$, p. 236.

Trois états de la traduction, no 3, p. 236.

Troisième niveau, $\mathrm{n}^{\circ} 4$, p. 379.

Troupeau de référence, $n^{\circ} 4$, p. 360 .

Truchement, $\mathrm{n}^{\circ} 1, \mathrm{p} .19$.

Turbine à gaz, no 3, p. 266

Tuyère, $n^{\circ} 3$, p. 261 . 
Uniformisation, $n^{\circ} 3$, p. 259

Unité de sens, $n^{\circ} 4$, p. 358.

Unité nutritive transformable, $n^{\circ} 4$, p. 363.

Unité phrasélogique, $\mathrm{n}^{\circ} 3$, p. 242.

Université d'Ottawa, $\mathrm{n}^{\circ} .4$, p. 405 .

Université de Montréal, no 4, p. 405.

UNT, no 4, p. 363.

Utilisation du fourrage, $n^{\circ} 4$, p. 361 .

Vache de remplacement, $\mathrm{n}^{\circ} 4$, p. 362

Vache gestante, $n^{\circ} 4$, p. 361 .

Vache gravide, $\mathrm{n}^{\circ} 4$, p. 361 .

Vache pleine, $n^{\circ} 4$, p. 361.

Valeur au livre, $n^{\circ} 4$, p. 360.

Valeur d'élevage, $n^{\circ} 4$, p. $360 ; n^{\circ} 4$, p. 361

Valeur de rachat, $n^{\circ} 4$, p. 362.

Valeur héréditaire, n०4, p. $360 ; n^{\circ} 4$, p. 361.

Valeur systémique, $\mathrm{n}^{\circ} 1$, p. 30.

Validation, test de, $n^{\circ} 2$, p. 167

Veau d'embouche, $n^{\circ} 4$, p. 361.

Veau d'engraissement, $n^{\circ} 4$, p. 361 .

Vecteur, $\mathrm{n}^{\circ} 4$, p. 378

Vêlage, $n^{\circ} 4$, p. 360.

Vêlage, taux de, $n^{\circ} 4$, p. 360

Verbalisation, $n^{\circ} 2$, p. 139.

Vérité de terrain, $n^{\circ} 4$, p. 378.

Vérité-terrain, $n^{\circ} 4$, p. 378

Viande de bceuf, no 4, p. 359.

Visée, angle de, $\mathrm{n}^{\circ} 4$, p. 366.

Visée latérale, radar à, $n^{0} 4$, p. 374

Visualisateur, $n^{\circ} 4$, p. 378 .

Visualisation, $n^{\circ} 4$, p. 378.

Visualiser, $n^{\circ} 4$, p. 378.

Visualiseur, $n^{\circ} 4$, p. 378

Visuel, $n^{\circ} 4$, p. 378.

Vitesse de l'orateur, no 3 , p. 207.

Vocabulaire technique, $\mathrm{n}^{\circ} 3$, p. 199.

Vouloir-dire d'un locuteur, $\mathrm{n}^{\circ} 4$, p. 358.

Voyoute, no 3 , p. 288.

WEIDNER, n 3, p. 275.

Xénisme, nº 2, p. 122.

Zootechnicien, $n^{\circ} 4$, p. 360 . 


\section{Index anglais des mots et des sujets traités}

Actual change, no 3, p. 265 .

Adiabatic, no 3, p. 262.

Aerospace Dictionary, no 4, p. 398

Agency, n०2, p. 155.

Al-Ahram Establishment, n०2, p. 157.

ALECSO, $\mathrm{n}^{\circ} 2$, p. 156.

ALPS, no 4, p. 403.

Analysis phase, $\mathrm{n}^{\circ} 2$, p. 130 .

Arab Academy, n०2, p. 158

Arab Organization for Agricultural Development, $n^{\circ} 2$, p. 157.

Arab Standardization and Metrology Organization, $\mathrm{n}^{\circ} 2$, p. 157.

Arab university, n०2, p. 158

Arab world, $\mathrm{n}^{\circ} 2$, p. 155 .

Arabic Language Academy, n० 2, p. 155.

Arabic reference, $n^{\circ} 2$, p. 155

Arabization, $\mathrm{n}^{\circ} 2$, p. 155

Arabization conference, $\mathrm{n}^{\circ} 2$, p. 158.

Argumentative discourse, $\mathrm{n}^{\circ} 3$, p. 227.

ASMO, n०2, p. 156.

Aural system, no 4 , p. 309.

Australia, no 2, p. 186.

Automated Language Processing System, no 4 , p. 403.

Availability factor, $\mathrm{n}^{\circ} 3$, p. 266.

Bangla, no 3, p. 212

Baudelaire, no 3, p. 217.

Behaviour, no 3, p. 226

Bridging, n० 3, p. 212.

Brinkmann, Karl-Heinz, no 4, p. 397.

Cairo Academy, no 2, p. 155.

Carburetion, external, no 3, p. 272

Carburetion, internal, n०3, p. 272.

Card-in-slot mechanism, no 2, p. 130.

Carnot fraction, $n^{\circ} 3$, p. 265.

Carrier, third level, $\mathrm{n}^{\circ} 4$, p. 379.

Catford, $n^{\circ} 4$, p. 345 .

Checking phase, $n^{\circ} 2$, p. 132

Close translation, n० 3 , p. 212.

Coded slot, $n^{\circ} 2$, p. 130 .

Cognitive psychologist, $n^{\circ} 1$, p. 68

Communicative competence, $\mathrm{n}^{\circ} 3$, p. 211.

Commuter, no 4 , p. 379 ; n० 4 , p. 380 .
Commuter air carrier, no 4 , p. 379.

Commuter air carrier operator, no 4 , p. 379

Commuter airline, $\mathrm{n}^{\circ} 4$, p. 379.

Commuter airlines, no 4 , p. 380 .

Commuter flight, no 4 , p. 382 .

Commuter industry, no 4 , p. 383 .

Commuter operator, no 4 , p. 379 .

Competence, $n^{\circ} 3$, p. 225.

Comprehension, $n^{\circ} 1$, p. $37 ; n^{\circ} 4$, p. 344.

Compression ignition engine, $n^{0} 3$, p. 272.

Conference interpreter, $n^{\circ} 1$, p. 91 .

Conference interpreting, $n^{\circ} 1$, p. $49 ; n^{\circ} 1$, p. 74.

Connector, n ${ }^{\circ} 4$, p. 379 .

Consecutive interpretation, no 1 , p. 37 .

Consecutive interpretation training, $n^{\circ} 2$, p. 148.

Consonant rhyme, no 4 , p. 310 .

Construction, $n^{\circ} 1$, p. 39 .

Court interpretation, $n^{\circ} 2$, p. 151

Courtroom, no 2 , p. 148.

Creative Writer's Handbook, no 3, p. 287.

Culture-specific, $\mathrm{n}^{\circ} 1$, p. 51 .

Data Systems Dictionary, no 4, p. 397.

Decoding, n० 3, p. $212 ; n^{\circ} 3$, p. 215.

Decoding translation, $\mathrm{n}^{\circ} 3$, p. 213 .

Deflagration, no 3 , p. 273.

Detonation, no $3, \mathrm{p} .273$

Diesel combustion, $n^{\circ} 3$, p. 273.

Discourse structure, no 3 , p. 230

Discourse the, $n^{\circ} 1$, p. 37.

Discourse, translation of non-literary, $n^{\circ} 3$, p. 217.

Displacement-type engine, no 3 , p. 267.

Distant translation, $n^{\circ} 3$, p. 212.

Dostert, no 1 , p. 75.

Dramatic verse, no 4 , p. 309.

Dryden, no 3 , p. 215.

Dual-task training, no 1, p. 97.

Dubber, no 1, p. 91.

Edinburgh Inventory of Handedness, $n^{\circ} 1$, p. 68 . Electric motor, no 3, p. 261.

Elsevier's Dictionary of Jewellery and Watchmaking, n' 4 , p. 396.

Encoding, no 3, p. 212.

Equilateral hyperbola, no 3, p. 263.

Error typology, no 1 , p. 106.

Escort interpretation, $\mathrm{n}^{\circ} 2$, p. 152.

Eurikon experiment, $\mathrm{n}^{\circ} 1$, p. 91 .

Exhausted, n०3, p. 263.

Expander, n० 3, p. 267

Expanding unit, no 3 , p. 267.

Expansion, no 3 , p. 261.

Expressional fluency, $n^{\circ} 4$, p. 330

External carburetion, no 3 , p. 272.

Federal Department of Immigration, $n^{\circ} 2$, p. 187.

Feedback loop, $\mathrm{n}^{\circ} 2$, p. 132 .

Feeder, no 4 , p. 379.

Feeder carrier, n० 4 , p. 381.

Feeding, no 4 , p. 381 . 
Flame front, $n^{\circ} 3$, p. 273.

Forget, C., n 4, p. 396.

Form, no 3, p. 214.

Formal competence, $n^{\circ} 3$, p. 211.

Formal module, $\mathrm{n}^{\circ} 1$, p. 106

Formal syntax, $\mathrm{n}^{\circ} 1$, p. 111.

Framework, $n^{\circ} 3$, p. 224.

Freelance work, $\mathrm{n}^{\mathrm{a}}$ 2, p. 190.

Fuel injection, $\mathbf{n}^{\circ} 3$, p. 272.

Functional activity, no 3, p. 224.

Gas turbine, $n^{\circ} 3$, p. 266.

Generalisation, no 1, p. 39

Generative linguistic, no 3 , p. 225.

Glossogenetic, $n^{\circ} 3$, p. 284.

Gounod, no 4, p. 316

Graphical notation, no 2, p. 129.

Half rhyme, $n^{\circ} 4$, p. 309.

Heat engine, $n^{\circ} 3$, p. 261

Heat pump, $n^{\circ} 3$, p. 261.

Hindi, no 3 , p. 212.

Hydraulic motor, no 3, p. 261.

I.c. engine, $n^{\circ} 3$, p. 268.

Ignition-controlled engine, $n^{\circ} 3$, p. 272.

Ignition system, $n^{\circ} 3$, p. 272.

Information processing approach, $\mathrm{n}^{\circ} \mathbf{1}, \mathrm{p} .37$.

Informative utterance, $n^{\circ} 3$, p. 228 .

Injection, fuel, $n^{\circ} 3$, p. 272 .

Injection system, $\mathrm{n}^{\circ} \mathrm{3}$, p. 273.

Input analyser, $\mathrm{n}^{\circ} 2$, p. 130.

Intended meaning, $\mathrm{n}^{\circ} 1, \mathrm{p} .108$.

Inter-cultural communication, $\mathrm{n}^{\circ} 1, \mathrm{p} .74$.

Inter-language reformulation, $\mathrm{n}^{\circ} 3$, p. 211 .

Inter-lingual communication, $\mathrm{n}^{\circ} 1, \mathrm{p} .74$.

Interactional, $n^{\circ} 3$, p. 226.

Interactive process, $n^{\circ} 2$, p. 129.

Internal carburetion, $\mathrm{n}^{\circ} 3$, p. 272

Internal combustion engine, $\mathfrak{n}^{\circ} 3$, p. 268.

Interpretation process, $\mathrm{n}^{\circ} 1$, p. 37 .

Interpreting, $\mathrm{n}^{\circ} 2$, p. 186

Interpreting strategy, $\mathrm{n}^{\circ} 1$, p. 109.

Iraqi Academy, $\mathrm{n}^{\circ} 2$, p. 156

Iraqi Scientific Academy, $\mathrm{n}^{\circ}$ 2, p. 156.

Isobaric, $\mathrm{n}^{\circ} 3$, p. 263.

Isothermal expansion, $\mathrm{n}^{\circ}$ 3, p. 263.

Jordanian Academy, no 2, p. 156.

Kinetic energy, no 3, p. 261

Kintsch and Van Dijk model, no 1, p. 37.

Knowledge, $n^{\circ} 3$, p. 226.

Knowledge of music, $n^{\circ} 4$, p. 318

Knowledge of prosody, $n^{\circ} 4$, p. 318.

Knowledge of rhyme, $n^{\circ} 4$, p. 318 .
Knowledge of vocal technique, $n^{\circ} 4$, p. 318 .

Kuwait Institute, $n^{\circ} 2$, p. 156 .

La Traviata, $n^{\circ} 4$, p. 311.

Language, $n^{0} 1$, p. 107.

Language transfer, $n^{\circ} 1$, p. 91.

Large regional, $n^{\circ} 4$, p. 380 .

Laurentian University, $n^{\circ} 3$, p. 298.

Level, $n^{\circ} 4$, p. 379.

Linguistic context, $n^{\circ} 3$, p. 216 .

Linguistic methodology, $\mathrm{n}^{\circ} 3$, p. 224.

Linguistic universal, $\mathrm{n}^{\mathrm{O}} 1$, p. 109.

Linguistic variation, $\mathrm{n}^{\circ} 1$, p. 112

Listener, $\mathrm{n}^{\circ} 1, \mathrm{p} .51$.

Literary translation, $n^{\circ} 3$, p. 214.

Literature, translation of, $n^{\circ} 3$, p. 213.

Local, $n^{\circ} 4$, p. 380

Local airlines, $n^{\circ} 4$, p. 380.

Long-term memory, $\mathrm{n}^{\circ} 1$, p. 39

LSP discourse, no 3, p. 229.

Machine aids to translation, $n^{\circ} 4, p .403$.

Macroproposition, $\mathrm{n}^{\circ} 1$, p. 39.

Macrorule, $n^{\circ} 1$, p. 39.

Major, $n^{\circ} 4$, p. 380.

Mapping rule, $\mathrm{n}^{\circ} 1$, p. $38 ; \mathrm{n}^{\circ} 1$, p. 42.

Marathi, ${ }^{\circ}$ 3, p. 212.

Maximum theoretical efficiency, $n^{\circ} 3$, p. 265.

Meaning, $n^{\circ} 3$, p. 214.

Meaning, unit of, $n^{\circ} 1$, p. 37.

Mechanical energy, no 3, p. 261.

Medium regional, $\mathrm{n}^{\mathrm{0}} \mathbf{4}$, p. 380 .

Memory exercise, $n^{\circ} 1$, p. 97.

Mental framework, $n^{\circ} 2$, p. 129.

Mental process, $n^{\circ} 1$, p. 106.

Metaphrase, $n^{\circ} 3$, p. 215.

Microproposition, n ${ }^{\circ} 1$, p. 39.

Mozart, $\mathrm{n}^{\mathrm{0}}$ 4, p. 312

Music, knowledge of, no 4, p. 318.

National, $n^{\circ} 4$, p. 380.

National Accreditation Authority, n ${ }^{\circ} 2$, p. 187.

National Language Policy, $\mathrm{n}^{\circ} 2$, p. 188.

Nida, $n^{\circ} 4$, p. 346 .

Non-displacement type engine, $n^{\circ} 3$, p. 267.

Non-literary discourse, translation of, $n^{\circ} 3$, p. 217.

Nonverbal communication, $n^{\circ} 1$, p. 49.

Nonvocal communication, $\mathbf{n}^{\circ} 1$, p. 50.

Note-taking system, $n^{\circ} 2$, p. 150 .

Nozzle, $n^{\circ} 3$, p. 262.

Nuremberg trials, $\mathrm{n}^{\circ} 1, \mathrm{p} .74$.

OAPEC, no 2, p. 157.

Off-rhyme, no 4, p. 309.

OPEC, $\mathrm{n}^{\circ} 2$, p. 157.

Opera translator, no 4, p. 309.

Operational model, no 2 , p. 129.

Otto's cycle, no 3 , p. 266. 
Output synthesizer, no 2, p. 131.

Overseas professional qualifications, $\mathrm{n}^{\circ} 2$, p. 187.

Overstatement, $\mathrm{n}^{\circ} 3$, p. 213 .

P-v diagram, $\mathrm{n}^{\circ} 3$, p. 262.

Pan-Arab association, $n^{\circ} 2$, p. 157.

Pan-Arab organization, $n^{\circ} 2$, p. 156.

Paraphrase, $\mathrm{n}^{\circ} 3$, p. 215.

Paraphrasing, $n^{\circ} 1$, p. 97

Particularisation, $\mathrm{n}^{\circ} 1$, p. 39.

Physical quantity, n० 3, p. 261.

Pictograph, $n^{\circ} 2$, p. 149.

Polysemy, $n^{\circ} 4$, p. 344.

Polytropic, no 3, p. 263.

Pragmatic context, $n^{\circ} 3$, p. 216

Pressure energy, $n^{\circ} 3$, p. 261.

Pressure-volume diagram, $\mathrm{n}^{\circ} 3$, p. 262.

Prime mover, $n^{\circ} 3$, p. 268.

Production, $n^{\circ} 1$, p. 37

Prosody, knowledge of, $n^{\circ} 4$, p. 318.

Public speaking, $n^{\circ} 2$, p. 148.

Rapid combustion, no 3 , p. 273.

Ray, Punya Sloka, n 3, p. 214.

Receptivity, $\mathrm{n}^{\circ} 1$, p. 50.

Rectangular hyperbola, $\mathrm{n}^{\circ} 3$, p. 263.

Reencoding, $\mathrm{n}^{\circ} 3$, p. 215.

Reencoding translation, $n^{\circ} 3$, p. 213.

Reexpression, $\mathrm{n}^{\circ}$ 3, p. 211.

Reference, $n^{\circ} 3$, p. 216.

Reformulation, $\mathrm{n}^{\circ} 3$, p. 211.

Regional, no 4, p. 380 .

Regional air carrier, $n^{\circ} 4$, p. 381.

Regional carrier, n ${ }^{\circ} 4$, p. 381.

Repetition, $n^{\circ} 3$, p. 211.

Reproduction, $\mathrm{n}^{\circ}$ 3, p. 211.

Research institute, $\mathrm{n}^{\circ} 2$, p. 156.

Restructuring, $\mathrm{n}^{\mathrm{O}} 2$, p. 129

Restructuring phase, $n^{\circ} 2$, p. 130.

Reversible, $n^{\circ} 3$, p. 265.

Rhetorical, no 3, p. 224

Rhyme, knowledge of, $n^{\circ} 4$, p. 318.

Ring, $n^{\circ} 4$, p. 310 .

Sameness, $\mathrm{n}^{\circ} 1$, p. 111

SASO, no 2 , p. 157.

Satellite television, $n^{\circ} 1$, p. 91

Saudi Arabian Standardization Organization, $\mathrm{n}^{\circ} 2$,

$$
\text { p. } 157 .
$$

Scheduled air taxis, $n^{\circ} 4$, p. 380 .

Schleiermacher, $n^{\circ} 3$, p. 213.

Schmidt, Rudolf, $n^{\circ} 4$, p. 397.

Scientific data, $n^{\circ} 2$, p. 155 .

Scientific discourse, $n^{\circ} 3$, p. 229

Scientific terminology, $\mathrm{n}^{\circ} 2$, p. 155.

Screening potential interpreter, $\mathrm{n}^{\circ} 1$, p. 97.

Self-ignition, $n^{\circ} 3$, p. 271 .

Semantic unit, $n^{\circ} 2$, p. 130.

Sender, $\mathrm{n}^{\circ} 1$, p. 51.

Sense, $n^{\circ} 3$, p. 216
Set of relations, $\mathrm{n}^{\circ} 1$, p. 109.

Shadowing, $n^{\circ} 1$, p. 97.

Short, $n^{\circ} 4$, p. 381 .

Short-haul, no 4, p. 382.

Short-haul carrier, $n^{\circ} 4$, p. 381.

Short-term memory, no 1 , p. 39.

Signant, $n^{\circ} 3$, p. 211 .

Signate, $n^{\circ} 3$, p. 211

Simultaneous interpretation, $\mathrm{n}^{\circ} 1$, p. 37.

Small regional, $\mathrm{n}^{\circ} 4$, p. 380 .

Social interaction, $\mathrm{n}^{\circ} 1, \mathrm{p} .51$

Spark-ignited engine, no 3 , p. 272.

Specific speech function, $n^{0} 3$, p. 228.

Specification, $\mathrm{n}^{\circ} 1$, p. 39.

Speech Act Theory, no 3, p. 227.

Steiner, no 4, p. 346.

Stirling engine, $n^{\circ} 3$, p. 267.

Student reaction, $n^{\circ} 2$, p. 153 .

Style, no 3 , p. $214 ;$ no $^{\circ}$, p. 230

Stylistic issue, $n^{\circ} 3$, p. 224 .

Subjacency principle, $\mathrm{n}^{\circ} 1$, p. 111 .

Subtitler, $\mathrm{n}^{\circ} 1$, p. 91

Supplemental, $n^{\circ} 4$, p. 380.

Symbol, $n^{\circ} 2$, p. 149 .

Syntactic theory, $\mathrm{n}^{\circ} 1$, p. 106.

Syntax, $n^{\circ} 1$, p. 106.

Syrian Scientific Academy, n², p. 155.

Taber, no 4, p. 346.

Technical discourse, $\mathrm{n}^{\circ} 3$, p. $217 ; \mathrm{n}^{\circ} 3$, p. 229.

Technology, transfer of, $\mathrm{n}^{\mathrm{O}} 2, \mathrm{p} .155$.

Terminologist, $n^{\circ} 4$, p. 343.

Terminology, $\mathrm{n}^{\circ} 2$, p. $129 ; \mathrm{n}^{\circ} 2$, p. $130 ; \mathrm{n}^{\circ} 2$, p. 155.

Terminology of translation, $n^{\circ} 4$, p. 343.

Testing procedure, $\mathrm{n}^{\circ} 2$, p. 188 .

Textualization, $n^{\circ} 3$, p. 232.

The Abduction from the Seraglio, no 4, p. 312.

The Magic Flute, no 4, p. 309

Theoretic cycle, $\mathrm{n}^{\circ} 3$, p. 265.

Thermodynamic change, $n^{\circ} 3$, p. 261

Thermodynamic cycle, $\mathrm{n}^{\circ} 3$, p. 263.

Thermodynamic process, $n^{\circ} 3$, p. 261

Third level, $n^{\circ} 4$, p. $379 ; n^{\circ} 4$, p. 381.

Third level carrier, $\mathrm{n}^{\circ} 4$, p. 379.

Thought, $\mathrm{n}^{\circ} 1, \mathrm{p} .107$.

Three-phase model, $\mathrm{n}^{\circ} 1$, p. 51.

Three-phase operation, no 1 , p. 37.

Transactional, $\mathrm{n}^{\circ} 3$, p. 226.

Transfer of technology, $\mathrm{n}^{\circ} 2$, p. 155.

Transformulation, $\mathrm{n}^{\circ} 3$, p. 232 .

Translating, $\mathrm{n}^{\circ} 2$; p. 186 .

Translating for information, $n^{\circ} 2$, p. 135

Translating for publication, $n^{\circ} 2$, p. 133 .

Translating opera, $\mathrm{n}^{\circ} 4$, p. 309.

Translating Unit, $\mathrm{n}^{\circ} 2$, p. 187.

Translation, $n^{\circ} 4$, p. 343.

Translation models, $n^{\circ} 2$, p. 129

Translation of literature, $n^{\circ} 3$, p. 213.

Translation of Mozart/Schikaneder, $n^{\circ} 4$, p. 309.

Translation of non-literary discourse, $n^{0} 3$, p. 217.

Translation pedagogy, $\mathrm{n}^{\circ} 3$, p. 224.

Translation process, $n^{\circ} 2$, p. $129 ; n^{\circ} 2$, p. 135. 
Translation, terminology of, $n^{\circ} 4$, p. 343 .

Translator, $\mathrm{n}^{\circ} 1$, p. 91

Trunk, no 4, p. 380.

Tuning phase, $\mathrm{n}^{\circ} 2$, p. 131.

Tuning selector, $n^{\circ} 2$, p. 131 .

Understanding, $\mathrm{n}^{\circ} 2$, p. 129.

Understanding network, $n^{\circ} 2$, p. 130

Understanding phase, $\mathrm{n}^{\circ} 2$, p. 130.

Understatement, $\mathrm{n}^{\circ} 3$, p. 213 .

Unit of meaning, $n^{\circ} 1$, p. 37.

Urdu, no 3 , p. 212 .

Verbal codification, $\mathrm{n}^{\circ} 1$, p. 49.

Verdi, $n^{\circ} 4$, p. 311 .

Videotape, $n^{\circ} 2$, p. 148

Visual channel, $\mathrm{n}^{\circ} 1$, p. 50

Visual information, $\mathrm{n}^{\mathrm{o}} 1, \mathrm{p} .51$.

Vocal technique, knowledge of, $n^{\circ} 4$, p. 318

Voice-over artist, n० 1, p. 91.

Wagner, $n^{\circ} 4$, p. 310

Weak rhyme, $n^{\circ} 4$, p. 309.

Working fluid, $n^{\circ} 3$, p. 261

Working memory, $\mathrm{n}^{\circ} 1$, p. 39

World knowledge, $n^{\circ} 3$, p. 226. 